\title{
Stretchable and transparent hydrogels as soft conductors for dielectric elastomer actuators
}

\section{Citation}

Chen, Baohong, Yuanyuan Bai, Feng Xiang, Jeong-Yun Sun, Yong Mei Chen, Hong Wang, Jinxiong Zhou, and Zhigang Suo. 2014. "Stretchable and Transparent Hydrogels as Soft Conductors for Dielectric Elastomer Actuators." J. Polym. Sci. Part B: Polym. Phys. 52 (16) (June 16): 10551060. doi:10.1002/polb.23529.

\section{Published Version}

doi:10.1002/polb.23529

\section{Permanent link}

http://nrs.harvard.edu/urn-3:HUL.InstRepos:13919153

\section{Terms of Use}

This article was downloaded from Harvard University's DASH repository, and is made available under the terms and conditions applicable to Open Access Policy Articles, as set forth at http:// nrs.harvard.edu/urn-3:HUL.InstRepos:dash.current.terms-of-use\#OAP

\section{Share Your Story}

The Harvard community has made this article openly available.

Please share how this access benefits you. Submit a story.

\section{Accessibility}




\title{
Stretchable and transparent hydrogels as soft conductors for dielectric elastomer actuators
}

\author{
Baohong Chen ${ }^{1, \#}$, Yuanyuan Bai ${ }^{2, ~ \#, ~ F e n g ~ X i a n g ~}{ }^{2}$, Jeong-Yun Sun ${ }^{3}$, Yongmei Chen 4 , \\ Hong Wang ${ }^{2, a)}$, Jinxiong Zhou ${ }^{1, a)}$ and Zhigang Suo ${ }^{3}$
}

${ }^{1}$ State Key Laboratory for Strength and Vibration of Mechanical Structures, International Center for Applied Mechanics and School of Aerospace, Xi'an Jiaotong University, Xi'an 710049, China ${ }^{2}$ School of Electronics and Information Engineering, Electronic Materials Research Laboratory, Xi'an Jiaotong University, Xi'an 710049, China

3School of Engineering and Applied Sciences, Kavli Institute of Bionano Science and Technology, Harvard University, Cambridge, Massachusetts 02138, USA

4School of Science, MOE Key Laboratory for Non-Equilibrium Synthesis and Modulation of Con densed Matter and Department of Chemistry, Xi'an Jiaotong University, Xi'an 710049, China

A soft ionic conductor can serve as an artificial nerve in an artificial muscle. We synthesize a polyacrylamide hydrogel containing a hygroscopic salt, lithium chloride. Two layers of the hydrogel are used as ionic conductors to sandwich a dielectric elastomer and fabricate a highly stretchable and transparent actuator. When the two layers of the hydrogels are subject to a voltage, the actuator reduces its thickness and expands. An areal strain of $134 \%$ is demonstrated. We calculate the voltage-strain curves using a model that accounts for the elastic constraint of the hydrogel and the inhomogeneous deformation of the actuator. For actuators fabricated with the hydrogel of various thicknesses and with the dielectric elastomer of various prestretches, we find excellent agreement between experimental data and theoretical predictions.

\# Equal contribution;

a) hwang@mail.xjtu.edu.cn, jxzhouxx@mail.xjtu.edu.cn 


\section{Introduction}

When a soft dielectric sandwiched between two soft conductors is subject to a voltage, electric charges of the opposite polarities accumulate on the faces of the dielectric, causing the dielectric to reduce thickness and expand area. Such an electromechanical transducer mimics the function, but not the anatomy, of a muscle. The technology is under intense development for broad applications, including soft actuators, ${ }^{[1-3]}$ bio-inspired soft robotics, ${ }^{[4]}$ tactile and haptic interfaces, ${ }^{[2,5]}$ adaptive optics, ${ }^{[6-8]}$ generators, ${ }^{[9-10]}$ morphing wings, ${ }^{[11]}$ airships, ${ }^{[12]}$ and vibration isolators. ${ }^{[13]}$

In most demonstrations, the soft conductors are made of carbon grease, which is very compliant and exerts nearly no constraints to the deformation of the dielectric. The disadvantages of carbon grease are also obvious. ${ }^{[14]}$ The opaqueness of the grease limits its applications where transparency is needed, such as tunable optics. ${ }^{[7]}$ Inevitable abrasion and friction deteriorate the performance of the conductors. It is desirable to replace carbon grease with soft, transparent, elastomeric conductors.

One way to realize an elastomeric conductor is to disperse conducting materials, such as carbon powders, carbon nanotubes, carbon nanowires and graphene flakes, into an elastomer. This approach gives rise to conductors with some unique feature such as self-healing properties. ${ }^{[15]}$ However, compared to low modulus of dielectric elastomer, typically tens of $\mathrm{kPa}$, the stiffness of such elastomeric conductors, say 910kPa for PDMS with carbon black, ${ }^{[16]}$ is high and will constrain the deformation of DE actuator markedly. In addition, the dispersed 
carbon-based materials render the conductors opaque. Other stretchable conductors under development include corrugated metal films, ${ }^{[14,17]}$ graphene sheets, ${ }^{[18,19]}$ silver nanowires, ${ }^{[20,21]}$ and carbon nanotube meshes. ${ }^{[22,23]}$ Using these approaches to realize highly stretchable and transparent conductors remains a challenge.

By contrast, many ionic conductors are highly stretchable and transparent, and can be used as soft conductors in dielectric elastomer transducers to achieve high-speed, large-strain electromechanical transduction without electrochemical reaction. ${ }^{[24]}$ For example, a hydrogel is a three-dimensional polymer network swollen with water. The polymer network provides the form of a soft solid, whereas water is an excellent ionic conductor. Recent advances have achieved hydrogels with stretchability over $2000 \%$ and toughness near $9000 \mathrm{~J} / \mathrm{m}^{2}{ }^{\left[{ }^{[25]} \text { Ionic }\right.}$ conductors are abundant and diverse: ample opportunities exist to develop ionic conductors to meet needs in specific applications. For instance, many hydrogels are biocompatible, and are suitable for biomedical applications. Hydrogels are also inexpensive and easy to make; they are ideal for demonstrating conceptual designs or studying electromechanical behavior. Hydrogels, however, dry out if water evaporates, and will be unsuitable for applications in the open air. We have recently synthesized a highly stretchable and transparent ionogel and demonstrated its use as nonvolatile, soft conductors in dielectric elastomer transducers. ${ }^{[26]}$

Here we synthesize a polyacrylamide hydrogel containing lithium chloride. Lithium chloride is used here because of its high solubility in water and its hygroscopic properties. At $25^{\circ} \mathrm{C}$ a saturated aqueous solution of lithium chloride is in equilibrium with the air of relative humidity of $11.30 \%$. [1967 Young see the paper in the dropbox folder] We then use the hydrogel as soft conductors, together with a commercially available dielectric elastomer VHB 4910 (3M), to fabricate dielectric elastomer actuators. A maximum area strain of $134 \%$ is demonstrated. We calculate the voltage-strain curves of the actuators by using a theoretical model that accounts for the 
constraint of the hydrogels. The experimental data of actuators made of the hydrogel of various thicknesses and the dielectric elastomer of various prestretches agree with theoretical predictions.

\section{Synthesis and experiment}

\subsection{Synthesis of hydrogel}

Acrylamide (AAm) monomer and lithium chloride ( $\mathrm{LiCl}$ ) powder were dissolved in deionized water. The concentration of AAm was set to be $2.2 \mathrm{M}$, and the concentration of LiCl was set to be and 1 8M. The crosslinking agent (N,N'-methylenebisacrylamide, MBAA), thermo-initiator (ammonium persulphate, APS) and accelerator (N,N,N',N'-tetramethylethylenediamine, TEMED), whose weight ratio were 0.06 wt.-\%, 0.17 wt.-\% and 0.125 wt.-\%, respectively, relative to acrylamide monomer, were subsequently added into the mixed solution. The uniformly mixed solution was transferred into a glass mould with dimensions 100.0 $\mathrm{mm} \times 100.0 \mathrm{~mm} \times \mathrm{t}(\mathrm{t}=0.3,0.5$ and 1.0mm $)$ and then gelled in an oven at $50{ }^{\circ} \mathrm{C}$ for 2 hours. The hydrogel is transparent and stretchable (Fig. 1).

\subsection{Electrical and mechanical characterization of hydrogel}

We used four-point method to measure the conductivity of the hydrogels. The conductivity increases with the concentration of LiCl. When the hydrogel was not stretched, the measured limiting molar conductivity of the hydrogels was about $70 \mathrm{Scm}^{2} / \mathrm{mol}$. This value may be compared with the limiting molar conductivity of aqueous solution of LiCl, which was 91 $\mathrm{Scm}^{2} / \mathrm{mol}$. Mechanical tests were performed using a tensile machine with a $100-\mathrm{N}$ load cell. The maximum rupture stretch of the hydrogel is 23 and the small-strain Young' modulus calculated 
at $10 \%$ strain is $1.8 \mathrm{kPa}$.

\subsection{Dielectric elastomer actuators using hydrogel as soft conductors}

We used the hydrogels as soft conductors to fabricate dielectric elastomer actuators

(Fig. 2). Layers of the hydrogel were synthesized in three thicknesses, $0.3 \mathrm{~mm}, 0.5 \mathrm{~mm}$ and 1.omm. They were cut into circular shape of diameter $20 \mathrm{~mm}$ by using a laser cutting system (Versa Laser VLS2.30, Universal Laser Systems). The dielectric used was the VHB 4910 (thickness 1mm, 3M). A membrane of the dielectric elastomer was prestretched radially $\quad p=2$, $2.5,3,3.5$ or 4 times its initial radius, and attached to a circular rigid frame of an insulating material and radius $r_{\mathrm{B}}=66 \mathrm{~mm}$. The central part of each face of the dielectric membrane was then attached with a circular layer of the hydrogel, which was connected through a thin line of the hydrogel to a metallic electrode fixed on the frame.

The working principle of the actuator is understood as follows (Fig. 2b) [2013 Keplinger]. The metallic electrode is an electronic conductor, whereas the hydrogel is an ionic conductor. When the voltage across the electrode/hydrogel interface is within a certain range $(\sim 1 \mathrm{~V})$, electrons and ions do not cross the interface, no electrochemical reaction occurs, and the interface forms an electrical double layer, like a capacitor. Charges in the electrode and in the hydrogel are separated over nanometers. By contrast, charges on the two faces of the dielectric are separated by its thickness (on the order of $0.1 \mathrm{~mm}$ in a stretched dielectric). Consequently, the electrical double layer has an enormous capacitance compared to the dielectric. When a high voltage is applied between the two electrodes, the voltage across the electrical double layer is much smaller than $1 \mathrm{~V}$, and nearly all the applied voltage drops across the dielectric. A small 
voltage across the electrical double layer prevents electrochemical reaction, and a large voltage across the dielectric enables electromechanical transduction. The charges of the opposite polarities on the two faces of the dielectric elastomer attract, so that the actuator reduces thickness and expands area.

The actuator was highly stretchable and transparent (Fig. 3). The voltage was applied between the two metallic electrodes at a ramp rate of $100 \mathrm{~V} / \mathrm{s}$. After each increment of voltage of $500 \mathrm{~V}$, a photograph of the deformed actuator was taken by using a digital camera. The recorded pictures were input into Photoshop software (Adobe $\mathrm{CS}_{5}$ ) and areal strain was calculated pixel-by-pixel. The areal strain was defined as areal $=\left(A-A_{p}\right) / A_{p}$, where $A$ is the area of the active region covered by the hydrogel for various voltages, and $A_{p}$ is the area of this region after prestretching. The adhesion between the hydrogel and the dielectric was adequate; no delamination was observed during the experiment.

\section{Theoretical model}

We calculate the voltage-strain behavior by using a theoretical model that accounts for the constraint of the hydrogels. Subject to a voltage, the active part of the actuator expands by a homogeneous deformation, but the surrounding annulus of the dielectric relaxes by an inhomogeneous deformation. We analyze this inhomogeneous deformation by adapting a method described in a previous paper. ${ }^{[27]}$ The difference is that here we need to add the two layers of hydrogels to the model.

Consider an actuator in several states (Fig.2). In the reference state, a circular dielectric 
membrane, radius $B$ and thickness $H_{0}$, is subject to no force and no voltage. A material particle, distance $R$ from the center, is marked by a filled square. A circular layer of a hydrogel, radius $A \lambda_{p}$ and thickness $H_{2} / 2$, is also stress-free. In the prestretched state, the dielectric membrane is subject to an equibiaxial prestretch, $\lambda_{p}$, and is attached to a circular rigid frame. The active region is prestretched to a circle of radius $A \lambda_{p}$ and then attached with the layers of the hydrogel of the same radius. The hydrogel in this state remains to be stress-free. The thickness of dielectric membrane is $H_{1}=H_{0} / \lambda_{p}^{2}$ and the combined thickness of the two layers of the hydrogel is $H_{2}$. In the actuated state, subject to a voltage, the thickness of the dielectric reduces to $h$ and the radius of the active region becomes $A \lambda$. Here $\lambda=r / R$ is the stretch of the active region, $r$ is the current coordinate of the material particle with reference coordinate $R$.

The actuator consists of an active region and a passive region. The passive region has only elastic energy due to deformation of dielectric elastomer, while the energy of the active region is attributed to the stretching of dielectric, stretching of the hydrogel and polarization of the dielectric. The hydrogel also contributes to the total volume of the active region. We use the Gent model ${ }^{[28]}$ to represent the elastic energy of the dielectric elastomer and hydrogel but with different shear modulus, $\mu_{i}$, and extension limit, $J_{\text {lim }}^{i}$, in which $i=\mathrm{DE}$ or Gel. The Gent model reads

$$
W_{i}^{\text {elas }}\left(\lambda_{1}, \lambda_{2}\right)=-\frac{\mu_{i} J_{\lim }^{i}}{2} \log \left(1-\frac{\lambda_{1}^{2}+\lambda_{2}^{2}+\lambda_{1}^{-2} \lambda_{2}^{-2}-3}{J_{\lim }^{i}}\right)
$$

where $\lambda_{1}=\frac{\partial r}{\partial R}$ and $\lambda_{2}=\frac{r}{R}$ are radial and hoop stretches, respectively. The energy density of the active region can be written as 


$$
W\left(\lambda_{1}, \lambda_{2}, E\right)=\Phi_{D E} W_{D E}^{\text {elas }}\left(\lambda_{1}, \lambda_{2}\right)+\Phi_{G e l} W_{G e l}^{\text {elas }}\left(\lambda_{1}, \lambda_{2}\right)+\Phi_{D E} W_{D E}^{\text {elec }}\left(\lambda_{1}, \lambda_{2}, E\right)
$$

where $\Phi_{D E}=\frac{H_{1}}{H_{1}+H_{2}}$ and $\Phi_{G e l}=1-\Phi_{D E}$ and $E$ is the electric field.

The deformation of the passive region is inhomogeneous and the corresponding nominal stresses, $s_{1}$ and $s_{2}$, can be evaluated readily by taking derivatives of Equation (1) with respect to $\lambda_{1}$ and $\lambda_{2}$,

$$
\lambda_{i} s_{i}=\lambda_{i} \frac{\partial W}{\partial \lambda_{i}}=\frac{\mu_{D E}\left(\lambda_{i}^{2}-\lambda_{1}^{-2} \lambda_{2}^{-2}\right)}{1-\left(\lambda_{1}^{2}+\lambda_{2}^{2}+\lambda_{1}^{-2} \lambda_{2}^{-2}-3\right) / J_{\lim }^{D E}}, i=1,2
$$

We assume the applied voltage is below the critical voltage for the onset of wrinkles, so that the deformation of the active region is homogeneous, $\lambda_{1}=\lambda_{2}=\lambda$ and $s_{1}=\mathrm{S}_{2}=s$. Assuming the ideal-dielectric model for the dielectric energy and evaluating derivative of Equation (2) with respect to $\lambda$, yields the expression for the stress of active region [16]

$$
\Phi_{D E} \lambda s+\Phi_{D E} \varepsilon E^{2}=\frac{\Phi_{D E} \mu_{D E}\left(\lambda^{2}-\lambda^{-4}\right)}{1-\left(2 \lambda^{2}+\lambda^{-4}-3\right) / J_{\lim }^{D E}}+\frac{\Phi_{G e l} \mu_{G e l}\left(\lambda^{2} \lambda_{p}^{-2}-\lambda^{-4} \lambda_{p}^{4}\right)}{1-\left(2 \lambda^{2} \lambda_{p}^{-2}+\lambda^{-4} \lambda_{p}^{4}-3\right) / J_{\lim }^{D E}}
$$

The Electric field, $E$, in Equations (2) and (4), are related to applied voltage $\psi$ via $E=\frac{\psi}{H_{0}} \lambda^{2}$

Since the deformation of the active region is homogeneous, the equilibrium condition is satisfied automatically. The deformation in the passive region is inhomogeneous. The force balance of the passive region dictates that

$$
\frac{\partial s_{1}}{\partial R}+\frac{s_{1}-s_{2}}{R}=0
$$

The boundary conditions are 


$$
\left.S_{1}\right|_{R=A}=\left.S\right|_{R=A},\left.\quad \lambda_{2}\right|_{R=A}=\left.\lambda\right|_{R=A} \text { and }\left.\lambda_{2}\right|_{R=B}=\lambda_{\text {pre }} \text {. }
$$

The boundary-value problem of Equation (5) and boundary conditions (6) are solved by using a shooting method. ${ }^{[27]}$

\section{Results and discussion}

The parameters used in our simulation are $\quad \mathrm{Gel}=0.6 \mathrm{kPa}, J_{\lim }^{D E}=\mathbf{2 5 3}$ and $J_{\lim }^{G e l}=500$. The relative permittivity of $\mathrm{DE}$ is set to be $\varepsilon=4.159 \times 10^{-11} \mathrm{~F} / \mathrm{m}$. The shear modulus $\mathrm{DE}$ of the dielectric membrane is found to lie in the range of $18 \sim 25 \mathrm{kPa}$. Figure 4 compares experimental results and theoretical predictions. The thicknesses of the hydrogel are set to be $0.3 \mathrm{~mm}, 0.5 \mathrm{~mm}$

and $1 \mathrm{~mm}$. Prestretches of the dielectric are imposed to be $\lambda_{p}=2,2.5,3,3.5$ and 4 . Markers are experiments and solid lines are the theoretical predictions. The experimental data agree well with the theoretical predictions. Both thickness of the hydrogel and the prestretches of the dielectric affect the voltage-strain curves. Large actuated strain is attainable under the conditions of large prestretch and thin hydrogels. For the polyacrylamide hydrogel containg $\mathrm{LiCl}$, at prestrech 4 and hydrogel thickness $0.3 \mathrm{~mm}$, an areal strain of $134 \%$ is attained.

\section{Conclusion}

We report that a polyacrylamide hydrogel containing lithium chloride can function as soft conductors for dielectric elastomer transducers. The hydrogel is ionically conductive, stretchable and transparent. Systematic studies, both experimental and theoretical, are conducted to investigate the influence of the thickness of the hydrogel and the prestretch of the 
dielectric. The model accounts for homogeneous deformation of the active region and the inhomogeneous deformation of the passive region. The theoretical prediction agree well with the experimental data. Areal strain up to $134 \%$ is demonstrated.

\section{ACKNOWLEDGEMENTS}

This research is supported by Natural Science Foundation of China (grants 61025002, 11072185 and 11021202). ZS acknowledges the support of NSF MRSEC (DMR-0820484) and visiting appointment at the International Center for Applied Mechanics. 


\section{References}

1. R. Pelrine, R. Kornbluh, Q. Pei and J. Joseph, Science, 2000, 287, 836-839.

2. F. Carpi, S. Bauer and D. De Rossi, Science, 2010, 330, 1759-1761.

3. I. A. Anderson, T. A. Gisby, T. G. McKay, B. M. OBrien and E. P. Calius, Journal of Applied Physics, 2012, 112, 041101-041120.

4. S. Kim, C. Laschi and B. Trimmer, Trends in biotechnology, 2013.

5. M. Matysek, P. Lotz and H. F. Schlaak, Tactile display with dielectric multilayer elastomer actuators, 2009.

6. F. Carpi, G. Frediani, S. Turco and D. De Rossi, Advanced Functional Materials, 2011, 21, 4152-4158.

7. S. Shian, R. M. Diebold and D. R. Clarke, Optics Express, 2013, 21, 8669-8676.

8. S. Döring, M. Kollosche, T. Rabe, J. Stumpe and G. Kofod, Advanced Materials, 2011, 23, 4265-4269.

9. J. Huang, S. Shian, Z. Suo and D. R. Clarke, Advanced Functional Materials, 2013.

10. R. D. Kornbluh, R. Pelrine, H. Prahlad, A. Wong-Foy, B. McCoy, S. Kim, J. Eckerle and T. Low, MRS bulletin, 2012, 37, 246-253.

11. D. P. Wang, J. D. Bartley-Cho, C. A. Martin and B. J. Hallam, Development of high-rate large-deflection hingeless trailing-edge control surface for the Smart Wing wind tunnel model, 2001.

12. C. Jordi, S. Michel and E. Fink, Bioinspiration \& biomimetics, 2010, 5, 026007.

13. R. Sarban, R. Jones, B. Mace and E. Rustighi, Mechanical Systems and Signal Processing, 2011, 25, $2879-2891$.

14. S. Rosset and H. R. Shea, Applied Physics A, 2013, 110, 281-307.

15. S. Michel, B. T. Chu, S. Grimm, F. A. Nüesch, A. Borgschulte and D. M. Opris, Journal of Materials Chemistry, 2012, 22, 20736-20741.

16. M. Bozlar, C. Punckt, S. Korkut, J. Zhu, C. Chiang Foo, Z. Suo and I. A. Aksay, Applied Physics Letters, 2012, 101, 091905-091907.

17. M. Benslimane, P. Gravesen, P. Sommer-Larsen, in Proc. SPIE 4695, Smart Structures and Materials (Ed: Y. Bar-Cohen), SPIE, San Diego, CA , 2002.

18. J. Zang, S. Ryu, N. Pugno, Q. Wang, Q. Tu, M. J. Buehler, X. Zhao, Nature Materials, 2013, $12,321$.

19. K. S. Kim, Y. Zhao, H. Jang, S. Y. Lee, J. M. Kim, K. S. Kim, J. -H. Ahn, P. Kim, J. -Y. Choi, B. H. Hong, Nature, 2009, 457, 706-710.

20. S. De, T. M. Higgins, P. E. Lyons, E. M. Doherty, P. N. Nirmalraj, W. J. Blau, J. J. Boland, J. N. Coleman, ACS nano, 2009, 3, 1767-1774.

21. S. Yun, X. Niu, Z. Yu, W. Hu, P. Brochu, Q. Pei, Advanced Materials, 2012, 24, 1321-1327.

22. L. Hu, W. Yuan, P. Brochu, G. Gruner, Q. Pei, Applied Physics Letters, 2009, 94, 161108

23. V. Scardaci, R. Coull, J. N. Coleman, Applied Physics Letters, 2010, 97, 023114

24. C. Keplinger, J.-Y. Sun, C. C. Foo, P. Rothemund, G. M. Whitesides, and Z. Suo, Science, 2013, 341, $984-987$.

25. J.-Y. Sun, X. Zhao, W. R. Illeperuma, O. Chaudhuri, K. H. Oh, D. J. Mooney, J. J. Vlassak and Z. Suo, Nature, 2012, 489, 133-136.

26. B. H. Chen, J. J. Lu, C. H. Yang, J. H. Yang, J. Zhou, Y. M. Chen, Z. Suo, Advanced Materials, 2014.

27. S. J. A. Koh, T. Li, J. Zhou, X. Zhao, W. Hong, J. Zhu and Z. Suo, Journal of Polymer Science Part B: Polymer Physics, 2011, 49, 504-515.

28. A. N. Gent, Rubber Chemistry and Technology, 1996, 69, 59-61. 


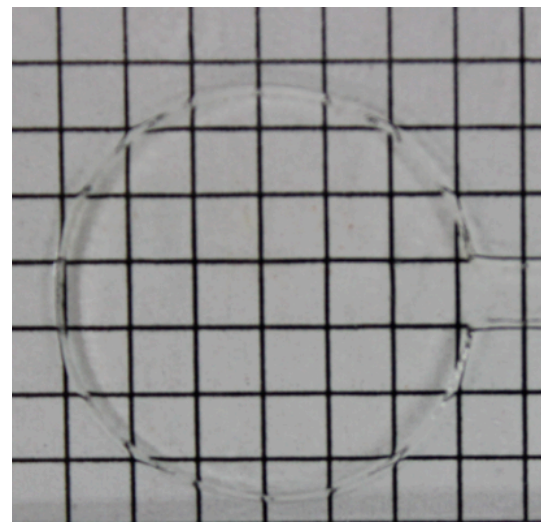

(a)

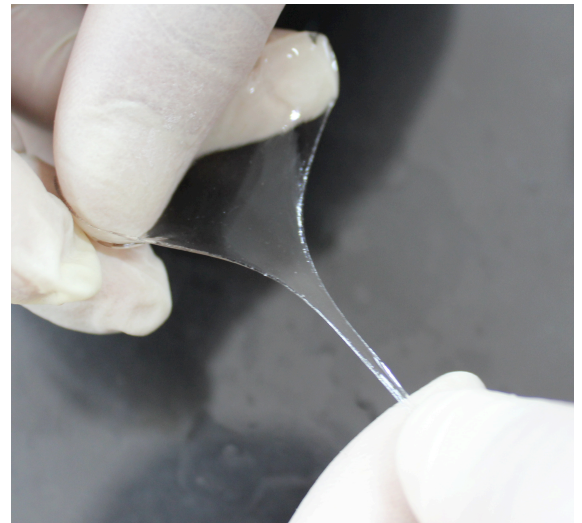

(b)

Figure 1. A polyacrylamide hydrogel containing lithium chloride. (a) The hydrogel is highly transparent. (b) The hydrogel is highly stretchable. 



Reference state



Prestretched state



Actuated state

(a)

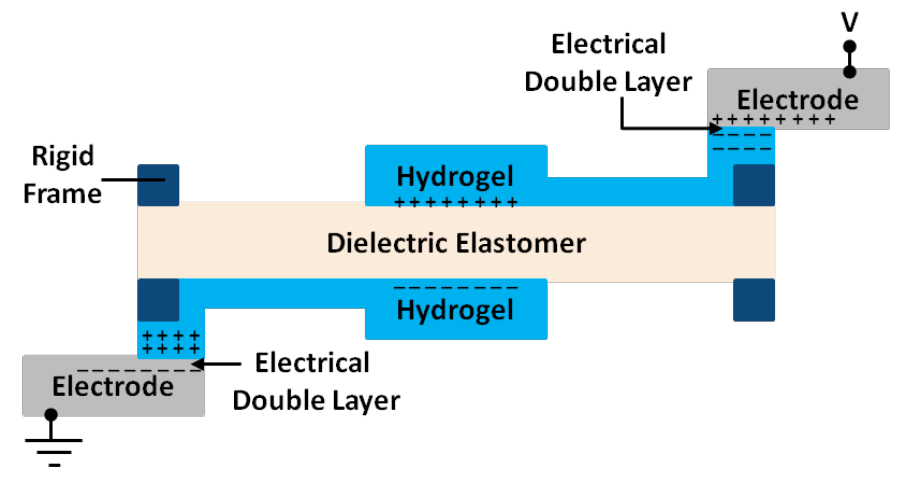

(b)

Figures 2. Schematic of an actuator made of a dielectric elastomer sandwiched between hydrogels. (a) Various states of the actuator. In the reference state, a circular membrane of a dielectric elastomer, radius $B$ and thickness $H_{0}$, and a circular membrane of a hydrogel, radius $A \lambda_{p}$ and thickness $0.5 H_{2}$, are both stress-free. A material particle is marked by coordinate $R$, and the radius of active region is $A$. In the prestretched sate, the dielectric elastomer is stretched radially by $\lambda_{p}$, and is attached to a rigid frame with radius $r_{\mathrm{B}}$. The thickness of the dielectric elastomer reduces to $H_{1}=H_{0} / \lambda_{p}^{2}$. The top and bottom faces of the dielectric elastomer are attached with layers of the hydrogel. In an actuated state, a 
voltage is applied the active region attains radius $A \lambda_{p}$ and thickness $h$, and the material particle moves to a place of radius $r$. (b) The side view of the experimental setup.

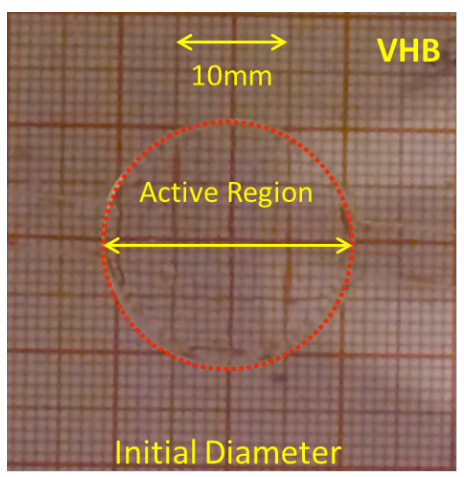

(a)

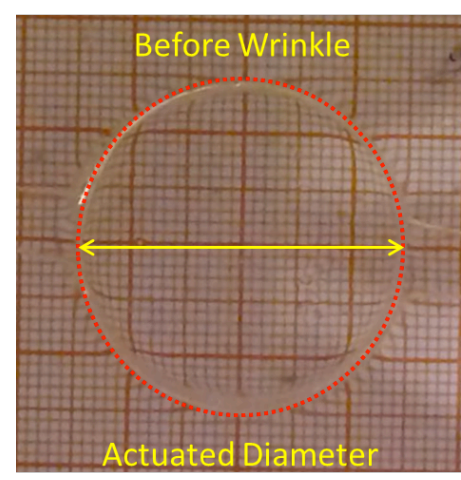

(b)

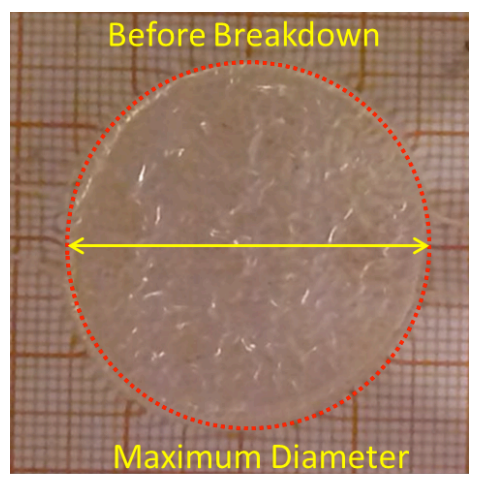

(c)

Figure 3. Voltage-induced deformation of a highly transparent actuator. (a) In the prestretched state, layers of the hydrogel of diameter $20 \mathrm{~mm}$ were attached on the prestretched dielectric membrane. The circumferential boundary of the active region is marked by the dotted line. (b) Actuated state before wrinkles set in. (c) Wrinkled state before electric breakdown. Maximum actuated deformation was attained in this state. 


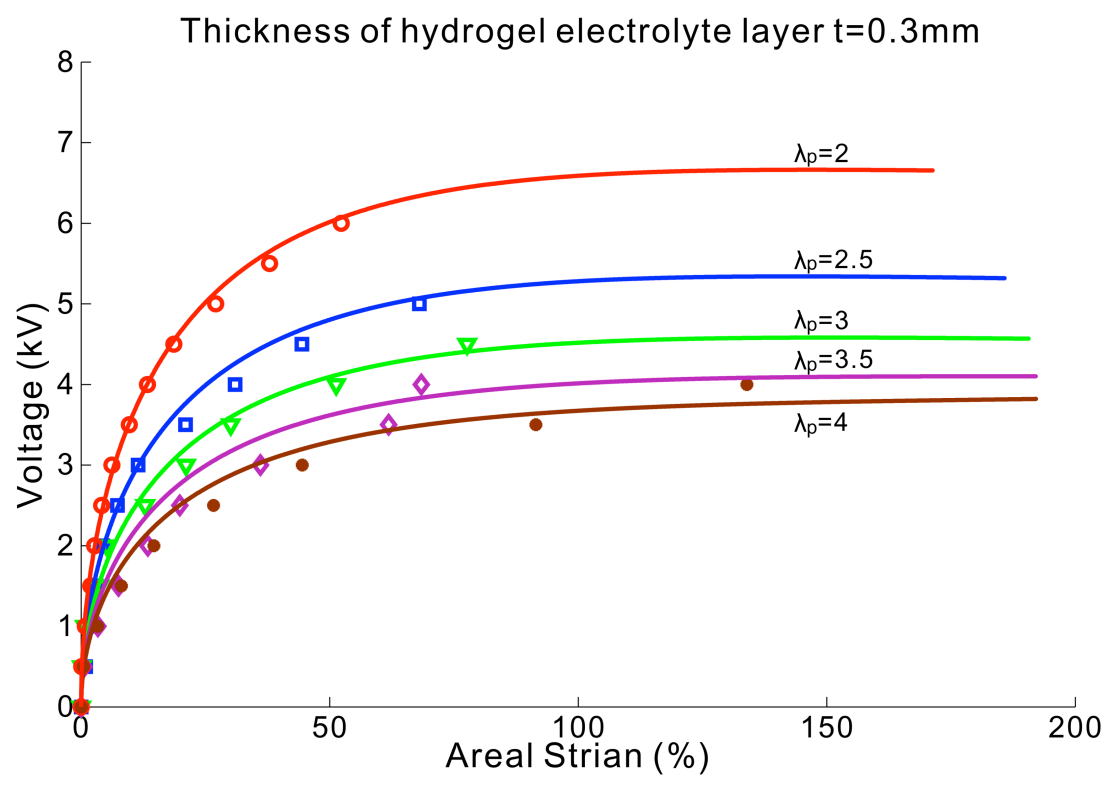

(a)

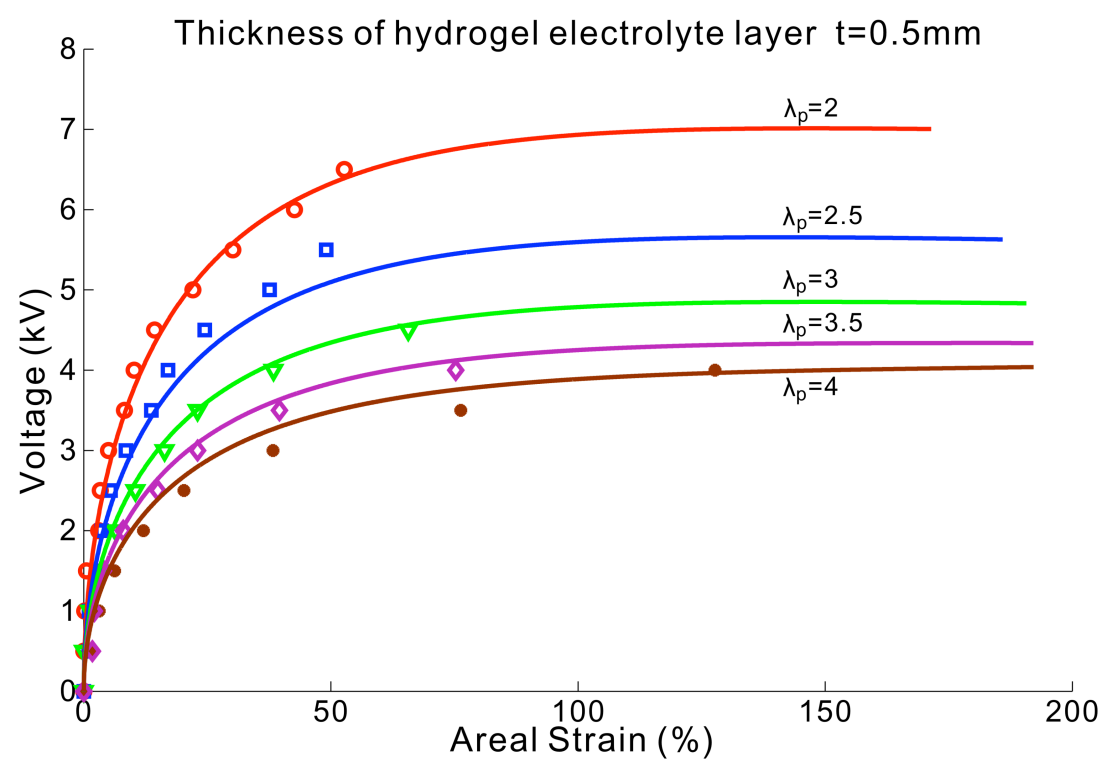

(b) 


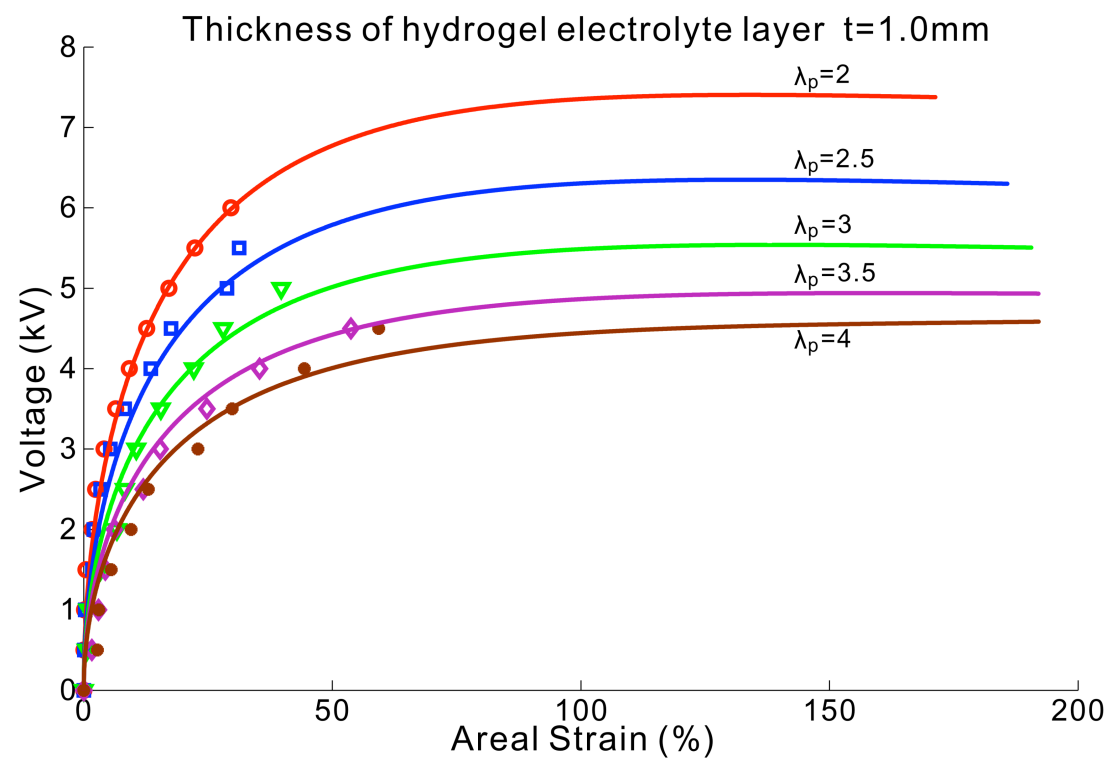

(c)

Figure 4. Comparison of measured and predicted voltage-strain curves with various thicknesses of hydrogel. Different levels of prestretches from $\lambda_{p}=2$ to $\lambda_{p}=4$ were imposed to the actuator. Markers denote experimental results while the solid lines represent model predictions. (a), (b) and (c) are the results for the hydrogel of thicknesses $0.3 \mathrm{~mm}, 0.5 \mathrm{~mm}$ and $1.0 \mathrm{~mm}$, respectively. 